Cahiers de recherches médiévales

\title{
Le réseau des écoles cathédrales dans la province ecclésiastique de Reims, dans la première moitié du XII ${ }^{\mathrm{e}}$ siècle
}

\section{Cédric Giraud}

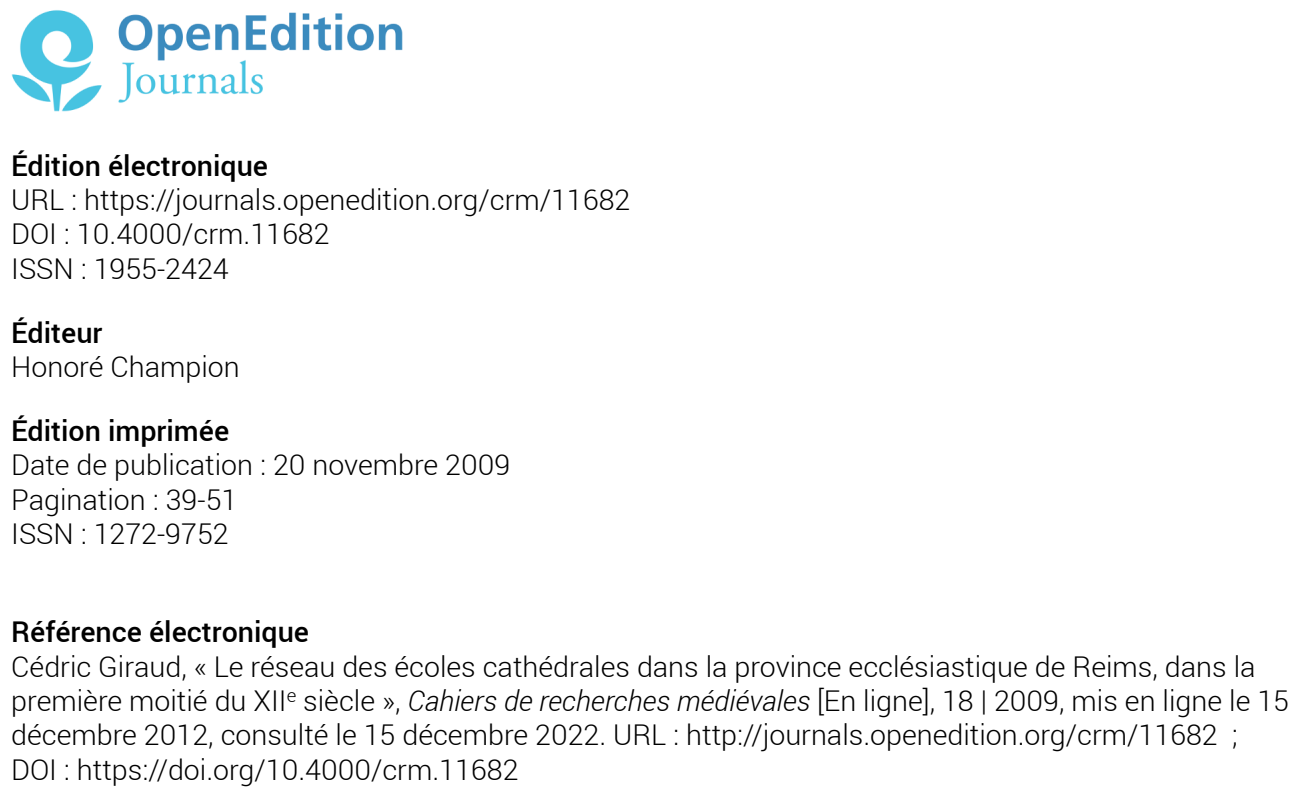

Tous droits réservés 


\title{
酶RM
}

\section{Le réseau des écoles cathéd rales dans la province ecclésiastique de Reims, dans la première moitié du $\mathrm{XII}^{\mathrm{e}}$ siècle}

\begin{abstract}
The chief original feature of the teaching practiced in the cathedral schools of the first half of the $12^{\text {th }}$ century was its loose institutional organization. Thus the concept of the network is a useful analytical tool which allows us to evaluate people's mobility. In the case of the the ecclesiastical province of Reims, the cathedral schools of Reims and Laon made up a network of schools that extended beyond the national borders, with exchanges between the two schools. The other cathedral schools are less well known and played a more modest role. Tracing the mobility of the scolares also involves observing the mobility of ideas within the society of the time: an examination of careerism and the circulation of writings shows us how the scholastics were responsible for the circulation of certain values. Thus we see how the cathedral schools, especially Laon, played a crucial role in the adoption of the Gregorian Reform by the clerics.
\end{abstract}

Résumé: L'originalité du système d'enseignement au sein des écoles cathédrales de la première moitié du XII siècle tient en grande partie à sa faible organisation institutionnelle. La notion de réseau constitue donc un apport non négligeable, car elle permet d'évaluer la mobilité des hommes. Dans le cas de la province ecclésiastique de Reims, les écoles cathédrales de Reims et de Laon constituent un réseau scolaire de portée internationale, avec des échanges entre les deux écoles. Les autres écoles cathédrales sont moins bien connues et ont joué un rôle plus effacé. La mobilité des scolares implique également celle des idées au sein de la société : le jeu des carrières et la transmission des écrits documentent la diffusion de valeurs par les milieux scolaires. Les écoles cathédrales, notamment celle de Laon, ont ainsi joué un rôle crucial dans l'adoption par les clercs de la réforme grégorienne.

Que ce soit comme étudiant ou comme professeur, Pierre Abélard a passé sa vie à voyager. Même en se limitant aux années antérieures à sa castration (1117 ou 1118), l'évocation donne le vertige. Né au Pallet, non loin de Nantes, en 1079, Abélard, poussé par le goût des études, quitte son pays natal et part étudier dans les écoles situées en France, sans doute Angers, Tours, Loches, puis Paris. De Paris, il va, sous la pression des événements, à Melun puis à Corbeil. Après un retour en Bretagne, il revient à Paris, puis à Melun avant de gagner Laon. Chassé par Anselme de Laon en 1113, il regagne Paris où il connaît la gloire, avant que de nouvelles tribulations ne le poussent à reprendre la route ${ }^{1}$. Cette itinérance, parfois choisie, souvent imposée, ne constitue pas un trait propre au seul Abélard, mais traduit plutôt la réalité des écoles du XII ${ }^{\mathrm{e}}$ siècle. De fait, en se focalisant sur cette époque, on est

\footnotetext{
${ }^{1}$ Pour une évocation plus détaillée, voir notamment J. Châtillon, «Abélard et les écoles », Abélard en son temps, Paris, 1981, p. 133-160, aux p. 134-149 et J. Verger, «De l'école d'Abélard aux premières universités ", Pierre Abélard. Colloque international de Nantes, éd. J. Jolivet et H. Habrias, Nantes, 2003, p. 17-28, aux p. 18-22.
}

Cahiers de Recherches Médiévales, 18, 2009 
assuré d'observer une vie scolaire alors en pleine expansion : parmi les éléments retenus traditionnellement pour caractériser la «Renaissance du XII siècle», on compte une véritable «révolution scolaire», c'est-à-dire l'augmentation significative des structures pédagogiques en même temps que l'amélioration, le renouvellement et la circulation accrue des savoirs transmis ${ }^{2}$. Dans les études portant sur les milieux scolaires du XII ${ }^{\mathrm{e}}$ siècle, l'historiographie a déjà bien rendu compte de cette dimension géographique, que ce soit au travers de monographies consacrées à un individu ou à des groupes identifiés par leur origine commune ou encore grâce à l'étude de centres scolaires dont l'importance a été justement jaugée à l'aune de leur rayonnement ${ }^{3}$.

Malgré le nombre et la qualité des travaux consacrés à ce point, il reste cependant encore utile d'évaluer le dynamisme des écoles du XII ${ }^{\mathrm{e}}$ siècle en croisant à la fois l'approche biographique et l'étude des différents centres scolaires. Appliquée à ces réalités, la notion même de « réseau », entendue simplement comme un ensemble de lieux et de personnes communiquant entre eux, peut s'avérer féconde dans la mesure où elle implique d'insister sur le phénomène de circulation considéré en tant que tel. L'étude d'un réseau scolaire est dès lors susceptible non seulement d'éclairer la carrière d'un maître, mais également d'indiquer les causes qui créent, par leur variété combinée, une géographie scolaire et hiérarchisent les lieux de savoir en centres plus ou moins attractifs. Parler de réseau suppose, en outre, de reconnaître à cette organisation une certaine unité qui lui donne sens, un fonds culturel commun par exemple. On ne peut alors esquiver la question de savoir si la culture partagée à laquelle le réseau scolaire donne accès consolide des stratégies familiales et plus largement une sociabilité, si elle favorise l'obtention de carrières dans et en dehors des écoles, en d'autres termes, si elle permet la constitution d'un réseau social. Il s'agit ainsi de déterminer la manière dont l'appartenance à un réseau scolaire détermine un mode d'action dans la société.

Pour séduisante que paraisse peut-être cette problématique, il faut d'emblée concéder que la réalisation de ce programme se heurte à des difficultés dont le rappel n'est pas pure clause de style. Elles tiennent tout d'abord à la nature même des écoles du XII ${ }^{\mathrm{e}}$ siècle. Au XII ${ }^{\mathrm{e}}$ siècle, pas plus d'ailleurs qu'à l'âge universitaire, il n'existe un type d'écoles uniformes qui partageraient des modes de fonctionnement et un cursus identiques. Les écoles cathédrales, retenues ici comme

\footnotetext{
${ }^{2}$ Sur les caractères de cette «renaissance », voir J. Verger, La renaissance du XII siècle, Paris, 1996.

${ }^{3}$ Sur le thème de la mobilité scolaire au XII ${ }^{\mathrm{e}}$ siècle, voir notamment É. Lesne, Histoire de la propriété ecclésiastique en France, t. 5, Les Écoles de la fin du VIII siècle à la fin du XII siècle, Lille, 1940, p. 464-467 et p. 524-525, A. L. Gabriel, Garlandia. Studies in the History of the Medieval University, le chapitre I «English Masters and Students in Paris during the Twelfth Century», Notre Dame - Francfort-sur-le-Main, 1969, p. 1-37, P. Classen, «La Curia Romana e le scuole di Francia nel secolo XII », Le istituzioni ecclesiastiche, I : Le istituzioni ecclesiastiche della societas christiana dei secoli XI-XII Papato, cardinalato e episcopato, Milano, 1974, p. 432-436, J. Ehlers, «Deutsche Scholaren in Frankreich während des 12. Jahrhunderts", Schulen und Studium im sozialen Wandel des hohen und späten Mittelalters, éd. J. Fried, Sigmaringen, 1986, p. 97-120, J. Verger, «La mobilité étudiante au Moyen Âge », Histoire de l'éducation, 50, 1991, p. 65-90, notamment les p. 66-68.
} 
objet d'étude, ont du moins pour point commun de dépendre institutionnellement du chapitre cathédral : cela ne préjuge en rien de leur uniformité et n'autorise pas non plus à taire que la vie scolaire a également existé au XII ${ }^{\mathrm{e}}$ siècle dans d'autres types d'établissements. Il suffit de rappeler l'existence d'écoles tenues par des moines, des chanoines réguliers ou encore des maîtres privés ${ }^{4}$. En outre, la documentation qu'ont laissée ces écoles cathédrales prend le plus souvent la forme de lettres empreintes de rhétorique, de traités anonymes et difficiles à dater ou de souscriptions peu explicites dans les chartes. Les sources ne livrent, par conséquent, qu'un témoignage qualitatif peu propice à la synthèse ou à la systématisation. C'est dire qu'il n'est pas question d'évaluer l'ampleur du phénomène réticulaire dans le monde scolaire, mais plutôt, à travers quelques études de cas, d'en préciser la qualité. Pour ce faire, le réseau des écoles cathédrales peut être étudié dans sa double dimension pédagogique et sociale.

\section{Le réseau pédagogique des écoles cathédrales}

Pour l'histoire scolaire du XII ${ }^{\mathrm{e}}$ siècle, où les synthèses sont plutôt des juxtapositions de monographies, il s'avère nécessaire de choisir un point d'observation précis pour ne pas donner de simples impressions. Le choix d'une province ecclésiastique comporte une part d'arbitraire dans la mesure où, comme on l'a rappelé, la vie scolaire ne se résume pas aux écoles cathédrales et où celles-ci ne bornent pas leurs rapports aux seules frontières provinciales. Ces indéniables limites sont cependant assez largement corrigées par le choix de la province ecclésiastique de Reims ${ }^{5}$. Dans la première moitié du XII ${ }^{\mathrm{e}}$ siècle, celle-ci connaît à la fois un renouveau scolaire remarquable, alors même que sa taille garantit un champ d'étude suffisamment vaste pour fournir des résultats pertinents. À l'aide de dépouillements personnels et de la bibliographie, ont été rassemblés des éléments documentant le réseau des écoles cathédrales de Reims et des onze évêchés suffragants (Amiens, Arras, Beauvais, Cambrai, Châlons, Laon, Noyon, Senlis, Soissons, Thérouanne et Tournai). En fonction de l'intensité des échanges observés, il est possible de distinguer une superposition de réseaux de type local, provincial et international.

L'indigence des renseignements que nous possédons sur la majorité des écoles cathédrales incite à les classer d'emblée parmi celles n'ayant qu'un rayonnement purement local. On en connaît à la rigueur les maîtres à la faveur des souscriptions présentes dans les chartes, mais aucun autre élément ne vient éclairer leur fonctionnement. À Senlis, la charge d'écolâtre n'est officiellement établie que dans la seconde moitié du XII ${ }^{\mathrm{e}}$ siècle, et l'on ne relève que le nom de maître Dreu

${ }^{4}$ Ce faisant, pour des raisons pratiques et non idéologiques, nous prolongeons une historiographie traditionnellement plus soucieuse des écoles cathédrales que des écoles monastiques; pour une rectification de cette tendance, voir S. N. Vaughn et J. Rubenstein, «Introduction », Teaching and Learning in Northern Europe, 1000-1200, éd. S. N. Vaughn et J. Rubenstein, Turnhout, 2006, p. 1-16.

5 Sur la géographie provinciale, voir, avec bibliographie, P. Demouy, Genèse d'une cathédrale. Les archevêques de Reims et leur Église aux XI et XII siècles, Langres, 2005, p. 427-437. 
dans une charte de 1133-11346. Même atonie à Amiens et Beauvais, où la consultation des chartes amiénoises de la première moitié du XII ${ }^{\mathrm{e}}$ siècle permet de retrouver le seul maître Rainier de Pinconio ${ }^{7}$, tandis que celles de Beauvais livrent les noms des maîtres Foulques et Godefroi en 1148. À Arras, les écolâtres connaissent des carrières plus remarquables, puisque trois d'entre eux deviennent archidiacres et que l'un de ceux-ci accède à l'épiscopat'. Enfin, l'existence d'une prébende destinée à faire vivre l'écolâtre explique sans doute les listes bien fournies pour la première moitié du XII ${ }^{\mathrm{e}}$ siècle à Soissons ${ }^{10}$, Noyon $^{11}$, Cambrai $^{12}$, Tournai $^{13}$ et Thérouanne ${ }^{14}$. Il faut donc supposer que l'obligation faite aux chapitres d'ouvrir une école et d'entretenir un écolâtre, comme le prévoyait notamment le concile romain de 1078, était diversement appliquée ou, à tout le moins, n'a pas suffi en bien des cas à créer une tradition scolaire significative. La vocation de ce type d'école locale consiste principalement à former les jeunes clercs à leur vie religieuse : l'éducation «professionnelle» ainsi dispensé ne suppose donc pas le recours à un maître connu

${ }^{6}$ É. Lesne, Histoire de la propriété, op. cit., p. 313-314.

${ }^{7}$ Acte 26 du Cartulaire du chapitre de la cathédrale d'Amiens, éd. J. Roux, t. 1, Amiens, 1905, p. 35-36.

${ }^{8}$ É. Lesne, Histoire de la propriété, op. cit., p. 314-315. Il faut aussi faire mention de maître Pierre en faveur duquel Eugène III demande une prébende à l'évêque de Beauvais en 1152, cf. $P L$ 180, 1498D-1499B.

${ }^{9}$ Cf. l'écolâtre Robert (1097-1113), devenu ensuite archidiacre d'Ostrevant (1116-1138), maître Hugues archidiacre (1145), et Frumaud, chanoine d'Arras, écolâtre (1142-1157), archidiacre d'Ostrevant (1157) puis évêque (1174-1183), voir les actes signalés par B.-M. Tock, Les chartes des évêques d'Arras (1093-1203), Paris, 1991, p. 389 et 360.

${ }_{10}$ Sur les écolâtres Bernard (1101), Hugues (1103-1107), Geoffroy (1110), Ingelramne (1117), Geoffroy (1122-1132), Hugues (1135), maîtres Gautier et Gilbert (1138), voir É. Lesne, Histoire de la propriété, op. cit., p. 311 et L. Carolus-Barré, «Les écoles capitulaires et les collèges de Soissons au Moyen Âge et au $\mathrm{XVI}^{\mathrm{e}}$ siècle», Enseignement et vie intellectuelle, Paris, 1975, p. 123-226, à la p. 128, repris dans Id., Études et documents sur l'Ile-de-France et le Picardie au Moyen Âge, t. 1, Compiègne et le Soissonnais, Compiègne, 1994, p. 363-467.

${ }^{11}$ Cf. l'écolâtre Garmunde (1102), l'écolâtre Foucher (1108-1119), Simon de Dorlenz (1115), maître Pierre (1132) et Robert (1155), cf. É. Lesne, Histoire de la propriété, op. cit., p. 320.

${ }^{12} \mathrm{Cf}$. les écolâtres Werinbold (fin $\mathrm{XI}^{\mathrm{e}}$ siècle - début XII ${ }^{\mathrm{e}}$ siècle), Odalric (1103), Géry (1113), Eustathius (acte entre 1116-1131, 1133), maîtres Mion et Gautier (1134), cf. É. Lesne, Histoire de la propriété, op. cit., p. 322-323.

${ }^{13}$ Cf. les écolâtres Garmunde (1101-1107), Hotfrid (1116-1121), Gautier III (1125-1126), Guerric d'Igny (1131-1136), maitres Letbert (1141) et Simon (1146-1149), cf. É. Lesne, Histoire de la propriété, op. cit., p. 336 et J. Pycke, Le chapitre cathédral Notre-Dame de Tournai de la fin $d u X I^{e}$ à la fin du XIII $I^{e}$ siècle. Son organisation, sa vie, ses membres, Louvain-la-Neuve - Bruxelles, 1986, p. 271-279.

${ }^{14} \mathrm{Cf}$. la série de maîtres: Otton (1112), Milon (1127), Hugues (1130) et Herman (1130-1133), Odon (1145-1146), cf. É. Lesne, Histoire de la propriété, op. cit., p. 332 ; sur l'écolâtre, voir l'étude à paraître de T. Kouamé : «La fonction d'écolâtre dans les écoles épiscopales de la période féodale $\left(\mathrm{X}^{\mathrm{e}}-\mathrm{XII}{ }^{\mathrm{e}}\right.$ siècle) ». Je remercie l'auteur de ce travail de m'y avoir donné accès avec libéralité. 
et n'oblige pas toujours à réserver une prébende à l'écolâtre, un chantre pouvant par exemple assurer facilement l'apprentissage du chant et des psaumes ${ }^{15}$.

La présence d'un évêque lettré ne change pas radicalement les conditions de fonctionnement de ce type d'école, comme l'illustre le cas de Châlons sous l'épiscopat de Guillaume de Champeaux (1113-1122). L'évêque a, semble-t-il, continué à enseigner la théologie et on possède même la trace de deux maîtres, Rainier et Étienne ${ }^{16}$. Un épisode célèbre de la Vita prima Bernardi de Guillaume de Saint-Thierry confirme la présence d'un nombre important de lettrés qui ont pour maître Étienne de Vitry ${ }^{17}$. Cependant, les magistri Rainier et Étienne font une carrière purement locale et rien n'indique qu'il en aille autrement pour leurs élèves. L'école de Tournai correspond également bien à ces écoles cathédrales d'importance locale: le cas tournaisien vérifie le fait que l'animation temporaire du réseau scolaire dépend principalement de la succession de maîtres remarquables ${ }^{18}$. En effet, l'arrivée d'un maître prestigieux comme Odon d'Orléans à la fin du $\mathrm{XI}^{\mathrm{e}}$ siècle donne à l'école un rayonnement international et attire des étudiants de toute la Chrétienté ${ }^{19}$. Mais la conversion rapide du maître à la vie régulière interrompt brutalement cette période faste. L'école cathédrale retrouve alors son rôle principalement local de formation du clergé : deux des quatre écolâtres alors connus sont d'anciens étudiants de l'école capitulaire ${ }^{20}$. Selon un schéma par ailleurs bien connu, la modestie d'une école locale, quelle que soit l'institution à laquelle elle s'adosse, pousse les élèves brillants ou aisés à gagner un centre plus important où ils complètent leur formation en se spécialisant dans une discipline. Ainsi Gautier de Mortagne, né vers le début $\mathrm{du}$ XII $^{\mathrm{e}}$ siècle dans une famille de châtelains liée à l'abbaye de Saint-Amand ${ }^{21}$, est-il d'abord offert dans sa jeunesse au chapitre d'Antoing. Il fait ses premières études à l'école cathédrale de Tournai, avant de se rendre aux écoles de Reims, puis de Laon,

\footnotetext{
${ }^{15}$ Voir les exemples indiqués par P. Delhaye, "L'organisation scolaire au XII ${ }^{\mathrm{e}}$ siècle », Traditio, 5, 1947, p. 211-268, aux p. 251-252, repris dans Id., Enseignement et morale au XII siècle, Fribourg - Paris, 1988, p. 1-58.

${ }^{16}$ Sur ce point, de nouveaux éléments m'ont été communiqués obligeamment par Charles de Miramon que je remercie, cf. son article sous presse: "Quatre notes biographiques sur Guillaume de Champeaux », Arts du langage et théologie aux confins des XI et XII siècles, éd. I. Rosier-Catach, Turnhout, à paraître.

${ }^{17}$ Cum enim episcopi gratia Catalaunum frequentaret, rediens aliquando traxit secum multitudinem nobilium et litteratorum, clericorum et laicorum. Quibus adhuc in domo hospitum demorantibus, dum novellas plantationes celestibus rigaret eloquiis, supervernit portarius monachus nuntians Stephanum de Vitreio, magistrum eorum, adesse ad renuntiandum seculo et cum eis commorandum. Guillaume de Saint-Thierry, Sancti Bernadi vita prima, 1, 13, PL 185, 263A.

${ }^{18}$ Voir la présentation de J. Pycke, Le chapitre cathédral Notre-Dame de Tournai, op. cit., p. 271-279.

${ }^{19}$ É. Lesne, Histoire de la propriété, op. cit., p. 522.

${ }^{20}$ Voir le tableau synthétique de J. Pycke, Le chapitre cathédral Notre-Dame de Tournai, op. cit., p. 278.

${ }^{21}$ L. Ott, Untersuchungen zur theologischen Briefliteratur der Frühscholastik, Münster, 1937, p. 126-128.
} 
où il se fixe et mène une carrière laonnoise qui le conduit à l'épiscopat (1155$1174)^{22}$.

Dans d'autres cas, l'école cathédrale paraît davantage insérée dans un réseau scolaire à l'échelle de la province. L'école cathédrale de Tournai ne répond pas tout à fait à cette définition dans la mesure où les scolares quittent le milieu local sans y revenir. En revanche, en d'autres cas, il est possible de suivre la mobilité scolaire entre écoles cathédrales, comme celles de Reims et de Laon sur deux générations (fin $\mathrm{XI}^{\mathrm{e}}$ du s. - années 1120$)^{23}$. La proximité géographique a d'évidence sa part dans le phénomène, ainsi que les rapports entre les différents maîtres tenant école dans les deux cités. L'hypothèse d'une formation à Reims d'Anselme de Laon auprès de Bruno de Cologne vient d'être relancée par Andrew B. Kraebel après examen des commentaires des Psaumes attribués à ces deux auteur $\mathrm{s}^{24}$. La tradition théologique laonnoise, dont on sait toute l'importance pour l'histoire de la sacra pagina au XII siècle, prendrait donc sa source dans l'une des écoles les plus fameuses de la fin du $\mathrm{XI}^{\mathrm{e}}$ siècle. En retour, l'école de Laon fournit des maîtres à sa voisine rémoise en la personne d'Albéric de Reims et de Lotulfe de Novare, anciens élèves d'Anselme de Laon, et dont l'enseignement reprend le flambeau anselmien dans les années 1120 sous les épiscopats de Raoul le Vert (1107-1124) et Renaud de Martigné (1124$1139)^{25}$. Durant la même décennie, le frère d'Anselme, Raoul († 1133), continue avec succès son propre enseignement à l'école cathédrale de Laon $^{26}$. Les deux écoles cathédrales entretiennent ainsi à l'échelon provincial un vivier de scolares.

Se nourrissant d'un réseau local et provincial, le rayonnement international d'une école a souvent été considéré comme un frein au développement des centres scolaires voisins. Le bonheur d'une école ferait ainsi le malheur des autres : dans le cas des écoles de Reims et de Laon, les seules à pouvoir revendiquer un statut international, et ce dès le $\mathrm{IX}^{\mathrm{e}}$ siècle, les choses sont sans doute plus complexes qu'une simple alternance d'apogée et de déclin. Il est ainsi notable que les échanges observés entre Reims et Laon, loin de freiner leur développement, semble étendre leur aire de rayonnement. Certes, le succès de l'école cathédrale de Laon sous

\footnotetext{
${ }^{22}$ Sur Gautier de Mortagne et les écoles, voir C. Giraud, «Per verba magistri. La langue des maîtres théologiens au premier XII ${ }^{\mathrm{e}}$ siècle ", Zwischen Babel und Pfingsten / Entre Babel et Pentecôte, Sprachdifferenzen und Gesprächsverständigung in der Vormoderne (8.-16. Jahrhundert). Différences linguistiques et communication orale avant la modernité (VIII $X V I^{e}$ siècle), éd. P. von Moos, Zurich - Berlin, 2008, p. 357-373, aux p. 360-363.

${ }^{23}$ Sur les écoles de Reims, voir J. R. Williams, "The Cathedral School of Rheims in the Eleventh Century », Speculum, 29, 1954, p. 661-677 et Id., «The Cathedral School of Rheims in the Time of Master Alberic, 1118-1136», Traditio, 20, 1964, p. 93-114, et sur Laon, C. Giraud, Per verba magistri. Anselme de Laon ( $\dagger$ 1117), son école et le mouvement théologique du XII siècle, thèse dactyl. Université de Paris IV Sorbonne, dir. J. Verger, Paris, 2006, à paraître en 2009 chez Brepols.

${ }^{24}$ Je remercie vivement Andrew B. Kraebel de m'avoir communiqué son article à paraître sous le titre: «Grammatica and the authenticity of the Psalms-commentary attributed to Bruno the Carthusian ».

${ }^{25}$ Voir J. R. Williams, "The Cathedral School of Rheims in the Time of Master Alberic », art. cit., et C. Giraud, Per verba magistri, op. cit., p. 530-534.

${ }^{26}$ Sur Raoul de Laon, voir C. Giraud, Per verba magistri, op. cit., p. 507-510.
} 
Anselme laisse quelque peu dans l'ombre sa voisine. Alors que rien de similaire n'est attesté à Reims à la même époque, on a pu repérer la trace précise de vingt-etun élèves ayant suivi les cours d'Anselme et sur lesquels nous possédons une mention significative : neuf sont originaires de France, dont trois de Bretagne, sept d'Angleterre, quatre d'Italie et au moins un de l'Empire ${ }^{27}$. De plus, l'analyse de la formation reçue auparavant par les différents étudiants confirme la valeur exemplaire du cas d'Abélard venu à Laon parachever sa formation par l'étude de l'Écriture sainte. C'est également le cas pour Robert d'Hereford, Hugues Métel, Matthieu d'Albano, Hugues d'Amiens et Guy Le Breton. Dans ces cas dont la diversité atteste qu'il ne s'agit pas d'exceptions, l'élève qui se rend à Laon a déjà entrepris l'étude des lettres. Selon les témoignages recensés, il a reçu, généralement dans sa famille ou dans une école locale, les rudiments de la formation latine et un enseignement de base dans les arts profanes. D'après les témoignages rassemblés, on vient à Laon pour être formé à la lecture de la Bible, non aux arts libéraux. Or, il est frappant de constater que dans la décennie 1120, les leçons de Raoul, comme celles d'Albéric de Reims, continuent d'attirer un auditoire international dans la tradition théologique anselmienne : on trouve à Reims des Anglais, des Allemands et des Lombards; à Laon surtout des Saxons, des Flamands et des Lotharingiens ${ }^{28}$. L'exemple de Gautier de Mortagne confirme que les passages d'une école à l'autre sont possibles. Force est également de reconnaître que, dans les deux villes, le phénomène connaît un temps d'arrêt dans la seconde moitié des années 1130: on connaît encore des maîtres à Laon et à Reims, mais des indices convergents suggèrent un rayonnement purement local ${ }^{29}$. Retenons en tout cas que la proximité géographique et la densité scolaire ne sont pas toujours synonymes de reclassement, au moins dans un premier temps.

Le réseau scolaire ainsi esquissé fait apparaître in fine deux types d'écoles cathédrales, celles qui assurent un enseignement de portée locale et celles promues au rang de centres internationaux influençant par ricochet une partie de la vie scolaire provinciale. Pour expliquer le phénomène, des facteurs d'ordre intellectuel sont à juste titre le plus souvent invoqués : des sources bien connues, et les historiens après elles, insistent sur la curiosité intellectuelle qui, confortée par le thème littéraire de l'exil scolaire, met en branle les scolares vers des maîtres prestigieux ${ }^{30}$. La présence plus ou moins durable des maîtres détermine ainsi une géographie scolaire par essence temporaire ${ }^{31}$. Des changements pédagogiques d'importance

\footnotetext{
${ }^{27}$ Pour le détail des cas, voir C. Giraud, Per verba magistri, op. cit., p. 73-108.

${ }^{28}$ Ibid., p. 510-523 et p. 530-534.

${ }^{29}$ «Manifestement, pendant ce temps, Reims vivait sur ses acquis et sur sa tradition, avec pour maîtres des produits du sérail », P. Demouy, Genèse d'une cathédrale, op. cit., p. 173.

${ }^{30}$ Cf. P. Delhaye, «L'organisation scolaire au XII ${ }^{e}$ siècle », art. cit., p. 240, P. Classen, «Die höhen Schulen und die Gesellschaft im 12. Jahrhundert», Archiv für Kulturgeschichte, 48, 1966, p. 155-180, aux p. 160-161, P. Johanek, «Klosterstudien in 12. Jahrhundert », Schulen und Studium..., op. cit. p. 35-68, aux p. 40-41.

${ }^{31}$ «Les maîtres se déplacent aussi souvent au gré des circonstances. Il paraît naturel qu'un écolâtre gouverne successivement plusieurs écoles. (...) L'enseignement devient aux $\mathrm{XI}^{\mathrm{e}}$ et $\mathrm{XII}^{\mathrm{e}}$ siècles une carrière, qui comporte déplacements et promotions; les maitres vont là où les
} 
expliqueraient même, dans certains cas, la faveur que connaissent certaines écoles au détriment d'autres ${ }^{32}$. Des arguments d'ordre géographique sont parfois également avancés: la concurrence entre écoles trop rapprochées, ainsi que les faibles possibilités de croissance urbaine constituent parfois des freins au développement scolaire $^{33}$.

La prise en compte plus large d'un contexte politique permet de compléter cette approche. Dans le cas des clercs anglais qui sont les mieux connus, on constate que le séjour à Laon fait partie d'une pratique sociale du voyage d'études sur le Continen $^{34}$. Il est effectué par des jeunes gens de bonne famille qui ont déjà reçu une formation insulaire et la complètent auprès de maître Anselme pour la théologie. L'existence de rapports scolaires étroits entre la ville de Laon et le monde anglonormand n'est pas pour surprendre, car des clercs d'origine anglaise ou soutenus par le roi d'Angleterre prennent une part active dans la vie laonnoise dès la fin du $\mathrm{XI}^{\mathrm{e}}$ siècle. Deux évêques de Laon contemporains d'Anselme sont liés aux rois d'Angleterre: l'évêque Élinand (1052-1096) a été chapelain puis ambassadeur du roi Édouard le Confesseur (1042-1066) à la cour de France, tandis que Gaudry (1106-1112) a occupé la charge de chancelier du roi d'Angleterre Henri I ${ }^{\text {er }}(1100-$ $1135)^{35}$. De plus, les rapports anglo-laonnois ne se limitent pas à la fonction épiscopale. Ils doivent s'entendre également de l'envoi de clercs formés à Laon en Angleterre: si de jeunes anglais viennent suivre des études à Laon, des chanoines de Laon sont également reçus avec honneur sur le sol anglais par d'anciens élèves d'Anselme ${ }^{36}$. De la même manière, les liens entre les écoles cathédrales de Laon et de Tournai s'inscrivent dans le contexte plus large de rapports entre les deux chapitres $^{37}$.

attirent les perspectives d'un renom à conquérir, d'une plus belle clientèle d'étudiants, d'un bénéfice plus satisfaisant », É. Lesne, Histoire de la propriété, op. cit., p. 467.

${ }^{32}$ Le conflit entre un «old learning » représenté par Anselme de Laon et un «new learning » typique d'un Pierre Abélard est ainsi vu par Stephen Jaeger comme un des facteurs explicatifs majeurs de l'histoire scolaire du XII ${ }^{\mathrm{e}}$ siècle, cf. The Envy of Angels, Cathedral Schools and Social Ideals in Medieval Europe (950-1200), Philadelphia, 1994, p. 229-236.

${ }^{33}$ R. W. Southern, Scholastic Humanism and the Unification of Europe, t. 1, Foundations, Oxford, 1995, tableau 1.

${ }^{34}$ C. Giraud, Per verba magistri, op. cit., p. 80-96.

35 Sur Élinand, voir S. Martinet, «Élinand, évêque de Laon méconnu (1052-1098)», Mémoires de la Fédération des sociétés d'histoire et d'archéologie de l'Aisne, 36, 1991, p. 58-78, et aussi A. Saint-Denis, Apogée d'une cité. Laon et le Laonnois aux XII et XIII siècles, Nancy, 1994, p. 83-85 et A. Dufour-Malbezin, Actes des évêques de Laon des origines à 1151, Paris, 2001, p. 15-16, et sur Gaudry, A. Saint-Denis, Apogée d'une cité, op. cit., p. 96-108.

${ }^{36}$ J. S. P. Tatlock, "The English Journey of the Laon Canons », Speculum, 8, 1933, p. 454465.

${ }^{37}$ Cf. Hériman de Tournai, Les miracles de sainte Marie de Laon, éd. A. Saint-Denis, Paris, 2008, p. 58-60. 


\section{Réseau social et transferts culturels}

Dès lors que l'on s'intéresse au rôle social joué par le réseau dans et hors les milieux scolaires, il convient de considérer ce réseau non pas uniquement comme un phénomène à expliquer, mais également comme un facteur explicatif à part entière. Pour être pertinent, ce dernier ne saurait se réduire à la constatation d'un simple passage dans les écoles cathédrales, c'est-à-dire à l'appartenance au groupe des litterati. La formation scolaire assure certes des compétences indispensables pour appartenir à un réseau scolaire comme la maîtrise d'une instruction " primaire », la capacité d'écrire et de lire le latin, ainsi que la connaissance partagée d'un certain nombre de textes de base. Pourtant, ce ne sont pas seulement des compétences acquises, mais également leur conscience revendiquée qui déterminent les contours du réseau scolaire. En effet, dans de nombreux cas, le passage par les écoles façonne une véritable identité dont les sources laissent clairement entendre le caractère affectif marqués ${ }^{38}$. Pour définir un réseau scolaire, la référence explicite à la figure magistrale ou au séjour dans les écoles constitue un indice de poids. À une époque où le cadre universitaire n'organise pas encore la vie scolaire, les liens affectifs développés par le maître avec ses élèves et par les élèves entre eux jouent un rôle primordial de lien social qui soude la communauté scolaire. Comme l'attestent les correspondances, les épitaphes ou les chroniques, l'amitié entre le maître et les élèves sert de cadre pour éduquer les scolares et de point de référence même après avoir quitté les écoles.

Parmi les écrits assurant la cohésion du réseau des scolares, les lettres ont été justement mises en valeur par John Van Engen comme des documents privilégiés ${ }^{39}$. Comme l'a bien montré cet historien, la lettre ne sert pas uniquement à mettre en scène l'amitié personnelle in absentia, mais fonctionne également comme signe de reconnaissance sociale. Elle peut même devenir un outil de communication au service du pouvoir qui met à profit les outils forgés dans le creuset scolaire. D'autres types de productions scolaires manifestent aussi l'attachement des scolares à leur milieu d'origine. L'étude des fonds médiévaux, que rendent possible les catalogues anciens de bibliothèques, aide ainsi à circonscrire l'aire d'influence d'une école et sa pénétration dans d'autres "communautés de savoir» comme les cloîtres. Le cas le plus typique est celui de Francon de Lobbes, qui est formé à Laon avant 1128 et devient abbé du célèbre établissement de 1149 à $1159^{40}$. La compétence acquise à Laon n'est pas une formation reçue seulement pour son abbaye d'origine car, selon l'auteur des Gesta abbatum, elle a également vocation à être dispensée à d'autres

\footnotetext{
${ }^{38}$ Voir les nombreux exemples cités et analysés par M. Münster-Swendsen, «The Model of Scholastic Mastery in Northern Europe c. 970-1200», Teaching and Learning in Northern Europe, 1000-1200, op. cit., p. 307-342, aux p. 308-318.

${ }_{39}$ J. Van Engen, «Letters, Schools, and Written Culture in the Eleventh and Twelfth Century", Dialektik und Rhetorik im früheren und hohen Mittelalter. Rezeption, Überlieferung und gesellschaftlische Wirkung antiker Gelehrsamkeit vornehmlich im 9. und 12. Jahrhundert, éd. J. Fried, Munich, 1997, p. 97-132.

${ }^{40}$ C. Giraud, Per verba magistri, op. cit., p. 518-523.
} 
établissements $^{41}$. On assiste ainsi à l'organisation d'un vrai réseau scolaire monastique qui prend sa source dans l'enseignement d'une école cathédrale : une grande abbaye envoie un ou plusieurs de ses moines doués se former dans un grand centre scolaire, pour ensuite faire profiter de cette remise à jour d'autres établissements de moindre importance. Il n'y a donc pas, en ce cas précis, séparation ni opposition entre le cloître et l'école, mais au contraire complémentarité et circulation des hommes, des connaissances et des manuscrits. En effet, comme nous le fait connaître le catalogue de Lobbes, l'abbaye s'ouvre, dans la première moitié du XII ${ }^{\mathrm{e}}$ siècle, aux nouveautés du temps, notamment aux livres bibliques glosés, qui constituent la contribution la plus durable de l'école de Laon à la théologie médiévale ${ }^{42}$.

En ce domaine, ce sont donc les écoles cathédrales les plus importantes qui fournissent la documentation permettant d'évaluer le moins mal les effets créés par leur fréquentation. Comme il faut s'y attendre pour la première moitié du XII ${ }^{\mathrm{e}}$ siècle, il demeure difficile d'établir des liens entre anciens élèves ou de prouver une corrélation entre la formation reçue et la carrière poursuivie. Les sources, quelque peu explicites qu'elles soient, laissent toutefois transparaître un intéressant phénomène social. Sur vingt-et-un élèves d'Anselme de Laon, seize accèdent à l'épiscopat, dont dix dans l'espace anglo-normand ${ }^{43}$. Il est évident que l'obtention de cet office explique l'intérêt des contemporains qui ont laissé des témoignages plus fournis que sur d'autres clercs aux carrières moins brillantes. Il serait cependant absurde de soutenir que le prestige reconnu à Anselme est resté sans influence pour ceux qui ont côtoyé le maître. Parmi d'autres, l'école cathédrale de Laon illustre donc le développement au XII ${ }^{\mathrm{e}}$ siècle de höhen Schulen assurant à leurs anciens élèves une place dans la société ${ }^{44}$. C'est le cas notamment du chanoine Guillaume de Corbeil qui est présenté par Henri $\mathrm{I}^{\mathrm{er}}$ aux moines de Cantorbéry en 1123 pour devenir archevêque de ce siège prestigieux. Contre la coutume, le roi d'Angleterre décide de faire élire à Cantorbéry un chanoine régulier et non un moine. Les chroniques du temps laissent entendre que le passé scolaire et religieux de

\footnotetext{
${ }^{41}$ Moris quippe Lobiensis ecclesie eatenus fuerat, ut non solum de suis aliorum locorum atque doctorum magisterio instruendos traderet, verum et de iis quoque et hoc studio plures et illustres habebat, alienis magistros accomodaret monasteriis, Gesta abbatum Lobbiensium, éd. W. Arndt, Hanover (MGH, Scriptores, 21), 1869, p. 331, 1. 5-8.

${ }^{42}$ F. Dolbeau, "Un nouveau catalogue des manuscrits de Lobbes aux $\mathrm{XI}^{\mathrm{e}}$ et XII ${ }^{\mathrm{e}}$ siècles », Recherches augustiniennes, 13, 1978, p. 3-36 et 14, 1979, p. 191-248, aux p. 17-18 et p. 194 ; sur les livres glosés, voir, avec bibliographie, l'excellente présentation d'A. Andrée, Gilbertus Universalis: Glossa ordinaria in Lamentationes Ieremie prophete. Prothemata et Liber I. A Critical Edition with an Introduction and a Translation, Stockholm, 2005, p. 7-35.

${ }^{43}$ C. Giraud, Per verba magistri, op. cit., p. 110-111.

${ }^{44}$ Des enquêtes précises portant sur la fin du XII ${ }^{\mathrm{e}}$ siècle ont cependant montré que la promotion sociale des magistri demeurait relativement limitée, du moins en France, cf. J. W. Baldwin: «Studium et Regnum: the Penetration of University Personnel into French and English Administration at the turn of the twelfth and thirtheenth centuries », Revue des études islamiques, 44, 1976, p. 199-215 et Id., «Master at Paris from 1179 to 1215 . A social Perspective", Renaissance and Renewal in the Twelfth Century, éd. R. L. Benson et G. Constable, avec C. D. Lanham, Cambridge Mass., 1982, p. 138-172 (rééd. Toronto, 1991).
} 
Guillaume explique le choix des moines : le séjour à Laon n'a pas été perdu et a donc aussi pesé dans l'élection à Cantorbéry ${ }^{45}$. La formation reçue crée donc un préjugé favorable pour la promotion de certains élèves à l'épiscopat. En effet, par rapport à d'autres pays comme la France ou l'Allemagne, le choix d'un futur évêque anglais dépend plus fortement qu'ailleurs de la formation reçue ${ }^{46}$. Comme on l'a vu, elle s'explique aussi par l'appartenance des élèves anglais aux milieux privilégiés de la cour et de l'Église. L'interaction entre l'ordre politique et intellectuel et leur mutuel soutien sont donc réels et ne souffrent pas une lecture purement politique ou intellectuelle.

Il faut également se demander si ce phénomène de promotion sociale ne s'accompagne pas d'un changement plus proprement religieux. Il s'agit, en d'autres termes, de déterminer si le passage dans une école cathédrale modifie l'appartenance d'un clerc à l'Église. Si l'on reprend l'exemple laonnois, il semble même que le réseau social soutienne la diffusion d'un idéal intellectuel et religieux prenant sa source à Laon. À chaque fois que les documents se font plus explicites, on note que les évêques formés par Anselme ont favorisé la réforme de l'Église et notamment la vie canoniale. Le point commun aux différents clercs anglais éduqués à Laon est leur attachement à la règle de saint Augustin et à la pratique d'une vie canoniale réformée revenant à la vie communautaire et à une discipline plus stricte. Guillaume de Cantorbéry, déjà mentionné, a été le premier prieur de la fondation canoniale de Saint-Osyth en Essex. Algar, évêque de Coutances de 1132 à 1151, et Robert, évêque d'Exeter de 1138 à 1155, ont également joué un rôle important dans la diffusion de la règle de saint Augustin, aussi bien en Angleterre qu'en Normandie ${ }^{47}$. Force est ainsi de constater que l'enseignement théologique d'Anselme rencontre des thèmes propres à la réforme ecclésiastique de son temps (conversion de l'homme intérieur, retour à la littera du texte biblique, question du transitus d'un ordre à un autre plus strict): bien que clerc séculier, Anselme développe une pastorale et une théologie fondées sur l'intériorité conçue comme un modèle pour la communauté chrétienne ${ }^{48}$. Dans ses sentences théologiques, le maître laonnois

\footnotetext{
${ }^{45}$ Cf. Symeonis monachi historia regum, éd. T. Arnold, Londres, 1885, § 206, p. 269 : necessitate ad unum illorum quatuor clericorum se conferunt, scilicet Willielmum de Curbellio, quem modestae vitae virum ac litteris bene eruditum familiarem habuerant, utpote cum venerandae memoriae archiepiscopo Anselmo saepissime ac familiariter conversatum. Le texte laisse supposer que Guillaume a été formé par Anselme de Cantorbéry alors que les sources laonnoises affirment qu'il a été éduqué à Laon, cf. Hériman de Tournai, Les miracles de sainte Marie de Laon, éd. cit., p. 168. L'ambiguité, qui ne saurait être une simple confusion dans des sources locales par ailleurs fort bien informées, prouve du moins l'existence d'un enjeu autour de la formation de l'archevêque.

${ }^{46} \mathrm{Cf}$. J. Barrow, «Education and the Recruitment of Cathedral Canons in England and in Germany, 1100-1225», Viator, 20, 1989, p. 117-138, aux p. 129-135.

${ }^{47}$ C. Giraud, Per verba magistri, op. cit., p. 84-85 et 88-90.

${ }^{48}$ Sur ce thème, voir la thèse inédite de S. R. Kramer, Secret Sins and the Privacy of Interior Homo in Twelfth-Century Theological Writings, Columbia University dissertation, 2002, dont certaines conclusions sont reprises dans S. R. Kramer et C. W. Bynum, «Revisiting the Twelfth-Century Individual. The Inner Self and the Christian Community », Das Eigene und
} 
entend ainsi réformer l'Église en façonnant aussi bien les intelligences que les cœurs $^{49}$. S'il n'est d'évidence pas nécessaire d'avoir entendu Anselme de Laon pour adopter les mots d'ordre de la réforme, il n'en demeure pas moins vrai que le maître a pu pousser certains de ses auditeurs à embrasser un état de vie réformé.

L'exemple de Robert de Béthune, évêque d'Hereford de 1131 à 1148, est particulièrement significatif $f^{50}$. Dans la biographie qui lui est consacrée, on apprend que le jeune clerc anglais a gagné la France en raison d'une vraie conversion intellectuelle: l'étude de la poésie, des arts et de la philosophie ne suffit plus à Robert qui veut pénétrer les secrets des choses saintes ${ }^{51}$. Il décide de se rendre à Laon en raison de la compétence et de l'orthodoxie d'Anselme. Le registre utilisé pour décrire la pratique pédagogique d'Anselme est essentiellement d'ordre moral. Cette formation culmine en effet dans des exercices, comme la prédication et la louange $^{52}$. Robert, à l'école d'Anselme, devient lui-même exemplaire

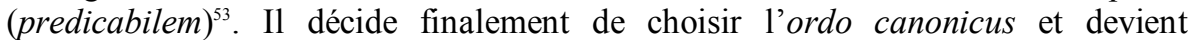
chanoine régulier à Lanthony avant 1115 , puis prieur dans les années 1120 . Une fois évêque, Robert d'Hereford apporte un soutien continu aux chanoines réguliers de son diocèse et mène une politique stricte de réforme morale en direction du clergé

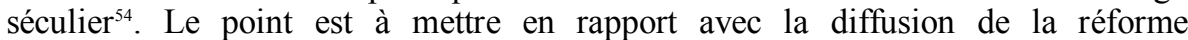
ecclésiastique depuis la province ecclésiastique de Reims : la présence régulière de papes grégoriens fuyant l'Italie, la fondation d'ordres nouveaux comme Prémontré au diocèse de Laon, le retour aux usages anciens chez les moines noirs, tous ces faits sont contemporains du rayonnement de l'école à $\mathrm{Laon}^{55}$. L'école cathédrale apparaît ainsi comme un instrument au service de la réforme religieuse qu'elle a contribué à diffuser efficacement.

\footnotetext{
das Ganze. Zum Individuellen im mittelalterlichen Religiosentum, éd. G. Melville et M. Schürer, Münster, 2002, p. 57-85.

${ }^{49}$ Sur ce point qui ne peut être développé dans le présent cadre, cf. C. Giraud, Per verba magistri, op. cit., p. 346-434.

${ }^{50}$ Voir J. Barrow, «Béthune, Robert de », Oxford Dictionary of National Biography, 5, 2004, p. 546-548 et C. Giraud, Per verba magistri, op. cit., p. 90-94.

${ }^{51}$ Guillaume de Wycombe, Vita Roberti episcopi Herefordiae, éd. Anglia sacra, t. 2, Londres, 1691, p. 300.

${ }^{52}$ Renuntians igitur his, hactenus animum convertit ad agiographa, fructum deinceps quaesiturus non folia. Parvi pendens autem quid de suo sensu garrire coepisset in divina pagina, quid disputare novella subtilitas, contulit se ad viros catholicos Willelmum Capellensem (sic), Anselmum Laudunensem, magistros emeritos quos documenta sanctorum patrum veraciter instituerant quid in divinis scripturis sentire, quid praedicare vel venerari deberent, Ibid..

${ }^{53}$ Ibid.

${ }^{54}$ Cf. J. Barrow, English Episcopal Acta, t. 7, Hereford 1079-1234, Oxford, 1993, p. 14-53 et Ead., "Clergy in the Diocese of Hereford in the Eleventh and Twelfth Centuries ", AngloNorman Studies, 26, 2003-2004, p. 37-53.

${ }^{55}$ Sur le contexte de réforme religieuse, voir Hériman de Tournai, Les miracles de sainte Marie de Laon, éd. cit., p. 105-114.
} 
L'originalité du système d'enseignement qui a été évoqué tient en grande partie à sa faible organisation institutionnelle. Des facteurs humains structurent très largement les écoles cathédrales, ce qui explique à la fois le petit nombre de sources et leur difficulté de traitement. Dans ce contexte, la notion de réseau constitue un apport non négligeable, car elle permet d'évaluer la mobilité des hommes en ne la réduisant pas à des stratégies individuelles arbitraires. Comme on a tenté de le montrer, la vitalité des écoles cathédrales peut s'expliquer par divers facteurs, aussi bien géographiques, intellectuels que politiques. Dans le cas de la province ecclésiastique de Reims, les écoles cathédrales de Reims et de Laon constituent un réseau scolaire de portée internationale, ce qui n'empêche pas les échanges entre les deux écoles. Les autres écoles cathédrales sont moins bien connues, ce qui s'explique sans doute par leur rôle beaucoup plus effacé à l'échelle provinciale. La mobilité des scolares implique également de suivre celle des idées au sein de la société : le jeu des carrières et la transmission des écrits documentent ainsi la diffusion de valeurs par les milieux scolaires. À l'époque considérée, la réforme religieuse n'est pas un enjeu extérieur aux écoles cathédrales. Les maîtres sont, en effet, des clercs qui ne sauraient rester indifférents aux normes promues par l'Église. Il apparaît ainsi que les écoles cathédrales, notamment celle de Laon, ont joué un rôle crucial dans l'adoption par les clercs de la réforme grégorienne.

Cédric Giraud

Université Nancy 2

ERL 7229 de Médiévistique 